\title{
Standard substances free quantification makes LC/ESI/MS non-targeted screening of pesticides in cereals comparable between labs
}

Wang, Tingting; Liigand, Jaanus; Frandsen, Henrik Lauritz; Smedsgaard, Jørn; Kruve, Anneli

Published in:

Food Chemistry

Link to article, DOI:

10.1016/j.foodchem.2020.126460

Publication date:

2020

Document Version

Peer reviewed version

Link back to DTU Orbit

Citation (APA):

Wang, T., Liigand, J., Frandsen, H. L., Smedsgaard, J., \& Kruve, A. (2020). Standard substances free quantification makes LC/ESI/MS non-targeted screening of pesticides in cereals comparable between labs. Food Chemistry, 318, [126460]. https://doi.org/10.1016/j.foodchem.2020.126460

\section{General rights}

Copyright and moral rights for the publications made accessible in the public portal are retained by the authors and/or other copyright owners and it is a condition of accessing publications that users recognise and abide by the legal requirements associated with these rights.

- Users may download and print one copy of any publication from the public portal for the purpose of private study or research.

- You may not further distribute the material or use it for any profit-making activity or commercial gain

- You may freely distribute the URL identifying the publication in the public portal 


\section{Journal Pre-proofs}

Standard substances free quantification makes LC/ESI/MS non-targeted screening of pesticides in cereals comparable between labs

Tingting Wang, Jaanus Liigand, Henrik Lauritz Frandsen, Jørn Smedsgaard, Anneli Kruve

PII: S0308-8146(20)30322-8

DOI: https://doi.org/10.1016/j.foodchem.2020.126460

Reference: FOCH 126460

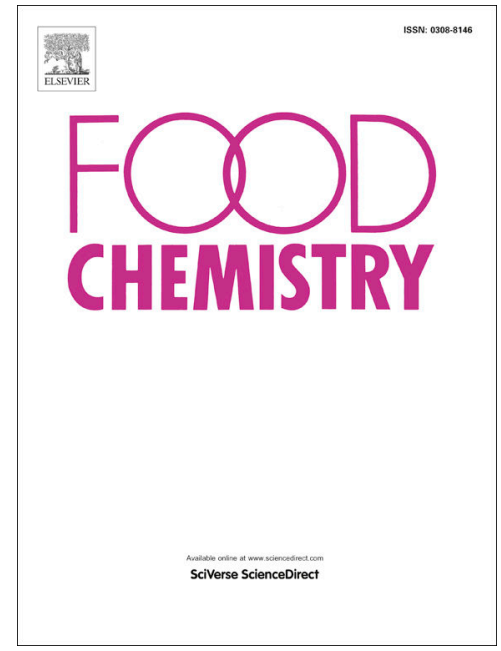

To appear in:

Food Chemistry

Received Date:

19 August 2019

Revised Date:

28 January 2020

Accepted Date:

19 February 2020

Please cite this article as: Wang, T., Liigand, J., Lauritz Frandsen, H., Smedsgaard, J., Kruve, A., Standard substances free quantification makes LC/ESI/MS non-targeted screening of pesticides in cereals comparable between labs, Food Chemistry (2020), doi: https://doi.org/10.1016/j.foodchem.2020.126460

This is a PDF file of an article that has undergone enhancements after acceptance, such as the addition of a cover page and metadata, and formatting for readability, but it is not yet the definitive version of record. This version will undergo additional copyediting, typesetting and review before it is published in its final form, but we are providing this version to give early visibility of the article. Please note that, during the production process, errors may be discovered which could affect the content, and all legal disclaimers that apply to the journal pertain.

(C) 2020 Published by Elsevier Ltd. 


\section{Standard substances free quantification makes LC/ESI/MS non-targeted screening of pesticides in cereals comparable between labs}

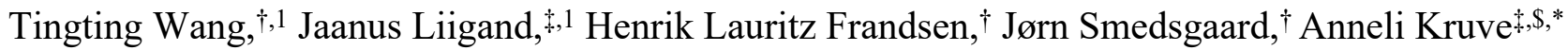

† National Food Institute, Research Group for Analytical Food Chemistry, Technical University of

Denmark, Kemitorvet Building 202, Kgs. Lyngby, DK-2800, Denmark

$\ddagger$ University of Tartu, Institute of Chemistry, Ravila 14a Tartu, 50411, Estonia

\$ Department of Environmental Science and Analytical Chemistry, Stockholm University, Svante Arrhenius väg 16, 10691 Stockholm

KEYWORDS. Quantification without standards; Pesticides; LC/ESI/MS; Food monitoring; Cereal

* Corresponding author: Anneli Kruve

E-mail address: anneli.kruve@ut.ee

${ }^{1}$ These authors contributed equally to this work 


\section{ABSTRACT}

LC/ESI/MS is the technique of choice for qualitative and quantitative food monitoring; however, analysis of a large number of compounds is challenged by the availability of standard substances. The impediment of detection of food contaminants has been overcome by the suspect and non-targeted screening. Still, the results from one laboratory cannot be compared with the results of another laboratory as quantitative results are required for this purpose. Here we show that the results of the suspect and non-targeted screening for pesticides can be made quantitative with the aid of in silico predicted electrospray ionization efficiencies and this allows direct comparison of the results obtained in two different laboratories. For this purpose, six cereal matrices were spiked with 134 pesticides and analysed in two independent labs; a high correlation for the results with the $R^{2}$ of 0.85 .

KEYWORDS: Quantification without standards; Pesticides; LC/ESI/MS; Food monitoring; Cereal 
1

2

3

4

5

6

7

8

9

\section{INTRODUCTION}

LC/ESI/MS is widely used for qualitative and quantitative analysis in food monitoring. This technique allows for the detection of a wide range of compounds such as pesticides, mycotoxins, veterinary drugs, other contaminants, and their metabolites in various food products (Dzuman, Zbynek, Zachariasova, Milena, Veprikova, Godula, \& Hajslova, 2015; Jamin, Bonvallot, \& Tremblay-franco, 2014; Mol et al., 2008). In ESI source, different compounds possess vastly different ionization efficiencies, the number of gas-phase ions produced at the same molar concentration; therefore, the quantification of these compounds requires corresponding standard substances to establish the relation between MS response and analyte concentration (Hermans, Ongay, Markov, \& Bischoff, 2017; Kruve, Kaupmees, Liigand, \& Leito, 2014). For example, more than 1000 different compounds against pests are in use worldwide (EU - Pesticides database; Li \& Jennings, 2017). Thus, it is common that pesticide residue analysis includes 500 or more analytes in the routine scope and the same number of standard substances needs to be purchased and included in the sequence. Unfortunately, standard substances are often unavailable for various reasons and it is even more of a predicament when new active substances are put on the market, residue definitions are changed to include metabolites of compounds or when compounds that are newly discovered or unstable.

Non-targeted analysis and suspect screening earn much attention in food safety monitoring in these years, as these allow identifying compounds present in the sample from MS data without the need for authentic standard substances (Fraser et al., 2014; Hird, Lau, Schuhmacher, \& Krska, 2014). However, for quantification purposes, standard substances are still required. As numerous tentatively identified compounds are often found it becomes either impossible or too expensive to purchase all these tentatively identified compounds. Furthermore, the peak areas obtained for different samples can be compared only if run in the same sequence and no matrix effect is expected. Comparison between different days, instruments or laboratories is impossible. Therefore, the ability to obtain quantitatively comparable results that do not depend on the instrument and lab is of utmost interest and importance (Kruve \& Kaupmees, 2017).

To overcome this problem some semi-quantification approaches have been proposed. An approach based on chemical similarity was proposed by, eg., Kalogiouri et al.,(Kalogiouri, Aalizadeh, \& 
Thomaidis, 2017) who selected several most appropriate standard substances for semi-quantification according to the similarity calculation of molecular descriptors between tested molecules and quantification standard substances (14 phenolic compounds). Pieke et al., (Pieke, Granby, Trier, \& Smedsgaard, 2017) demonstrated a proof of concept strategy for semi-quantification using LC/ESI/MS combining generic ESI ion source optimization using 18 quantification markers where the marker with the smallest difference in retention time was chosen to give the best average prediction of the concentration of the analytes. However, due to the limited number of test compounds and lack of validation with more compounds, the prediction quality is still unclear.

The prediction of ionization efficiency (IE) has the potential to offer higher accuracy and a wider application range. Recent attempts to predict ESI response have considered multiple physiochemical parameters simultaneously, and a correlation between ionization efficiency and different physicochemical properties (e.g., gas-phase basicity, $\log P$, polarity, $\mathrm{p} K_{\mathrm{a}}$, surface area, molar volume, etc.) has been observed (Alymatiri, Kouskoura, \& Markopoulou, 2015; Cramer, Johnson, \& Kamel, 2017; Fusaro, Mani, Mesirov, \& Carr, 2009; Golubović, Birkemeyer, Protić, Otašević, \& M., 2016; Hermans et al., 2017; Huffma, Poltash, \& Hughey, 2012; Oss, Kruve, Herodes, \& Leito, 2010; Wang et al., 2019; Wu et al., 2013). The application scopes have been confined to the specific type of compounds such as drug-like molecules (Cramer et al., 2017), amino acids (Hermans et al., 2017), peptides (Raji et al., 2009), steroids (Alymatiri et al., 2015), or organic acid et al (Wu et al., 2013). Unfortunately, these approaches have been hardly used for quantification. The solvent also has an important effect on ionization efficiency and needs to be taken into account, which is often neglected.

Our group (Kruve \& Kaupmees, 2017; Kruve et al., 2014; P. Liigand, Liigand, Cuyckens, Vreeken, \& Kruve, 2018; Oss et al., 2010) has previously proposed a more generic model to predict ionization efficiencies and the ionization efficiency predictions have been used to obtain the concentrations of small molecules analysis with gradient elution in ESI negative mode (Kruve \& Kaupmees, 2017) for the analysis of standard substances. Additionally, we have shown that ionization efficiencies can be predicted for compounds in case of the analysis of complicated matrices (blood, urine, etc.) with flow injection analysis (P. Liigand et al., 2018). Very recently we have developed a fast and easy Quantem approach to predict the ionization efficiencies for a wide range of compounds and to use it for estimating the concentration of the compounds in LC/HRMS non-targeted screening (J. Liigand et al., 2019). 
Based on the previously obtained promising results we aim to evaluate whether ionization efficiency prediction model can be used to reach quantitatively comparable results for suspect screening between labs for the analysis in food samples. Here we focus on the analysis of pesticides in cereals as cereals are one of the largest foods commodity and consumed worldwide (González-Curbelo, Herrera-Herrera, Ravelo-Pérez, \& Hernández-Borges, 2012), there is an intense attention to the occurrence of pesticides and mycotoxins exposure from cereal to maintain a high food safety standard. We, firstly, apply the Quantem model to predict the ionization efficiencies of detected pesticides to obtain the concentrations (using 4 orders of magnitudes spiking levels) in six common cereal matrices (oat, barley, rice, rye, wheat, and maize) (J. Liigand et al., 2019). Secondly, we evaluate the robustness of the concentration predictions of 134 common pesticides by comparing the results obtained from two laboratories in Denmark and Estonia on two different mass spectrometric setups (Agilent 6495 triple quadrupole and Bruker Daltonics micro-TOFq).

\section{MATERIALS AND METHODS}

\subsection{Chemicals and reagents}

Milli-Q grade water was used for all analyses $(18.2 \mathrm{M} \Omega \cdot \mathrm{cm}$, TOC 1-2 ppb, prepared by a Millipore Milli-Q Advantage A10 water-purification system). Acetonitrile (LC-MS grade), and formic acid (LC-MS grade) were purchased from Sigma-Aldrich (Steinheim, Germany). Magnesium sulfate (purity $>99 \%$ ) was purchased from Lach:ner (Neratovice, Czech Republic), and absorbent Bondesil-C18 was purchased from Agilent Technologies (Santa Clara, CA, USA). All other solvents used in this study were of analytical grade.

Thirty standard substances that were used to transfer the model from one lab to another were purchased from Sigma-Aldrich (Steinheim, Germany) or synthesized in-house (J. Liigand et al., 2019). All the pesticide standard substances (all with a purity $>96 \%$ ) were purchased from Dr. Ehrenstorfer (Augsburg, Germany). The pesticide standard substances stock solutions of $1 \mathrm{mg} / \mathrm{mL}$ were prepared in toluene and stored at $-18{ }^{\circ} \mathrm{C}$. A standard mixture of $10 \mu \mathrm{g} / \mathrm{mL}$ in $80 \%$ acetonitrile was prepared from these stock solutions. Working solutions in the concentration range of $4 \mathrm{nM}$ to $35 \mu \mathrm{M}$ on 6 different concentration levels were prepared by diluting the standard mixture with the extracts of blank cereal as a matrix (free of pesticide residues). Due to the instability of some of the compounds the same spiked 
samples could not be analyzed in two labs. To overcome this obstacle, the same sample preparation was carried out in both labs and the extracts were spiked. The small differences in the spiking experiments were determined by weighting and corrected thereafter. A full list of all the pesticides and compounds used for transferring the model (structures, retention time, isotope distribution, concentration, et al.) are shown in Table S1 in supporting information. The extracts used for the matrix matching were obtained by the extraction and clean-up procedure described below.

\subsection{Samples collection and sample extraction}

The blank samples (free of pesticides) of cereals specifically grown for preparation of European Union proficiency test material (EUPT) for the six EUPTs: EUPT-CF8 (wheat), EUPT-C3 (oat), EUPTCF10 (rye) and EUPT-C6 (barley), EUPT-CF9 (maize), EUPT-SRM6 (rice).

The extraction was based on the generic QuEChERS method developed by Dzuman's group (Dzuman, Zbynek et al., 2015). Two grams of homogenized cereal samples (wheat, oat, rye, rice, barley, maize) were weighed into a $50 \mathrm{~mL}$ polypropylene centrifugation tube, and $10 \mathrm{~mL}$ acidified Milli-Q water containing $0.2 \%$ formic acid was added and left to soak the matrix for $0.5 \mathrm{~h}$. Then, $10 \mathrm{~mL}$ acetonitrile was added, and the samples were extracted for $0.5 \mathrm{~h}$ using a laboratory shaker (Type 358S, Lubawa, Poland). Thereafter $4 \mathrm{~g}$ of magnesium sulfate and $1 \mathrm{~g}$ of sodium chloride were added, and the tube was shaken for $1 \mathrm{~min}$ followed by centrifugation (10 min, 4500 RPM; Centrifuge 5430R, Eppendorf AG, Hamburg, Germany). The organic upper layer $(2 \mathrm{~mL})$ was collected into a clean tube and shaken with $0.1 \mathrm{~g}$ of Bondesil-C18 and $0.3 \mathrm{~g}$ of magnesium sulfate for $2 \mathrm{~min}$ followed by centrifugation (10 $\mathrm{min}$, $4500 \mathrm{RPM}$ ). Then $1.5 \mathrm{~mL}$ of purified supernatant was transferred into an autosampler vial with insert and spiked with different concentration of tested compounds prior to injection on LC-MS system.

\subsection{Instrumental parameters}

\subsubsection{Agilent 6495 triple quadrupole}

Samples were analyzed on an Agilent 1290 ultrahigh performance liquid chromatograph (Agilent Technologies, CA, U.S.) coupled to an Agilent 6495 triple quadrupole instrument (QQQ) in University of Tartu (UT). Samples were injected onto an Agilent Zorbax RRHD SB-C18 reversed-phase column $(1.8 \mu \mathrm{m}, 2.1 \times 50 \mathrm{~mm})$. The mobile phase consisted of water $(\mathrm{A})$ containing $0.1 \%$ formic acid and acetonitrile (B). The analysis was done using a gradient elution at a flow rate of $0.3 \mathrm{~mL} / \mathrm{min}$ at $30{ }^{\circ} \mathrm{C}$. The gradient was from $5 \%$ to $100 \%$ of acetonitrile in $7 \mathrm{~min}$, then acetonitrile was maintained at $100 \%$ 
115 for $2 \mathrm{~min}$ and returned back to $5 \%$ of acetonitrile in $2 \mathrm{~min}$, and maintaining starting conditions at $5 \%$ of $116 \mathrm{~B}$ for 2 min equilibration with $5 \%$ of $\mathrm{B}$ to giving a total runtime of $13 \mathrm{~min}$.

117 An Agilent's Jet Stream electrospray ionization (ESI) interface was used in positive ion mode (ESI+) 118 with the following settings: capillary voltage $3 \mathrm{kV}$, nebulizer pressure $20 \mathrm{psi}$, sheath gas flow rate $11911 \mathrm{~L} / \mathrm{min}$, sheath gas temperature $350^{\circ} \mathrm{C}$, dry gas temperature $250{ }^{\circ} \mathrm{C}$, and dry gas flow rate $14 \mathrm{~L} / \mathrm{min}$. 120 Spectra were collected from $\mathrm{m} / z 100$ to $1100 \mathrm{Da}$. The injection volume was $1 \mu \mathrm{L}$. Peaks were manually 121 integrated, and peak areas were used for all calculations.

\subsubsection{Bruker Daltonics micro-TOFq}

Samples were also analyzed on an Agilent 1200 HPLC (Agilent Technologies, CA, U.S.) coupled to a Bruker Daltonics micro-TOFq (QToF) mass spectrometer (Bruker Daltonics, Bremen, Germany) in the lab of Technical University of Denmark (DTU). Samples were injected onto a Nucleoshell C18 reversedphase column $(2.7 \mu \mathrm{m}, 100 \times 2 \mathrm{~mm}$, MACHEREY-NAGEL GmbH \& Co. KG, Düren, Germany). The mobile phase consisted of water (A) containing $2.5 \mathrm{mM}$ ammonium formate at $\mathrm{pH}=3.0$, and acetonitrile (B). The analysis was done using a gradient elution at a flow rate of $0.3 \mathrm{~mL} / \mathrm{min}$ at $40{ }^{\circ} \mathrm{C}$ : isocratic at $2 \%$ of B for $1.0 \mathrm{~min}$, then increased to $100 \%$ of B at $22.0 \mathrm{~min}$, and maintained to $25 \mathrm{~min}$, whereafter the gradient returned to starting conditions $2 \%$ of B at 25.1 min followed by 2.9 min equilibration with $2 \%$ of B to giving a total runtime of $28 \mathrm{~min}$.

ESI+ was used in the following settings: capillary voltage $3 \mathrm{kV}$, nebulizer pressure $20 \mathrm{psi}$, dry gas temperature $200^{\circ} \mathrm{C}$, and dry gas flow rate $8 \mathrm{~L} / \mathrm{min}$. Funnel radio frequency $(\mathrm{RF}) 400 \mathrm{Vpp}$, multipole RF $200 \mathrm{Vpp}$. Spectra were collected from m/z 100 to $1100 \mathrm{Da}$ at a rate of $2 \mathrm{~Hz}$.

\subsection{Matrix effects, LoD, LoQ}

The matrix effect (ME) was calculated with the following formula in Eq. 1:

$$
\% M E=\left(\frac{\text { Slope }_{\text {matrices }}}{\text { Slope }_{\text {solvent }}}\right) \times 100 \%
$$

The calibration curves were prepared as the peak area versus the concentration of each analyte. Slope $_{\text {matrices }}$ is the slope of the calibration curves of analytes spiked into the cereal samples, Slope solvent $_{\text {is }}$ the slope of the calibration curves of analytes into the solvent. 


$$
\begin{array}{ll}
\text { LoD }=3.3 \times \frac{S_{\text {residuals }}}{\text { slope }} & \text { Eq. } 2 \\
L o Q=10 \times \frac{S_{\text {residuals }}}{\text { slope }} & \text { Eq. } 3
\end{array}
$$

142 Detection of limit (LoD) and detection of quantification (LoQ) were calculated based formula Eq. 2 143 and Eq. 3 (Evard, Kruve, \& Leito, 2016), $S_{\text {residuals }}$ is the residual standard deviation of the calibration

144 curve of each compound in the LoD region, and the slope is the slope value of the calibration curve of 145 the analytes.

\section{$146 \quad 2.5$ Model application.}

147 Previously developed Quantem model for predicting electrospray ionization efficiencies $(\log I E$, 148 predictions are made in the logarithmic scale) together with the transformation approach (J. Liigand et 149 al., 2019) was used to estimate the concentration of the compounds for the results of both labs. For this 150 empirical solvent descriptors (viscosity, surface tension, polarity index, $\mathrm{pH}, \mathrm{NH}_{4}$ presence) and 1444 151 PaDEL molecular descriptors were used for the model development (Yap, 2011). Solvent descriptors 152 were calculated based on the mobile phase used in the study and the retention time of each compound. 153 PaDEL descriptor calculator (Dong et al., 2015) was used to calculate the molecular descriptors of all 154 the compounds (http://www.scbdd.com/padel_desc/index/). For the transformation, the same compounds 155 as described previously (J. Liigand et al., 2019) were used.

156 For transferring the predicted ionization efficiency values, a set of compounds (either spiked to the matrix or as a standard solution) was measured in the linear range at the beginning and the end of the 158 sequence with the same method as analytes. From the analysis results, logarithmic response factors 159 $(\log R F)$ were calculated for transformation compounds as Eq. 4:

$$
\log R F=\log \frac{\text { Signal } \cdot I C}{c_{\text {spiked }}}
$$

Eq. 4

where Signal is the MS1 peak area and IC is an isotopic correction, which is the sum of the abundances of all isotopomers for each compound. Molar concentrations are used for calculation of response factor. 
163

164

165

166

167

168

169

170

171

172

173

174

175

176

177

178

179

180

181

182

Next ionization efficiencies were predicted using the Quantem model (J. Liigand et al., 2019). The generic ionization efficiency values predicted with Quantem model need to be transferred to the instrument and method-specific response factors. To obtain this the logarithmic response factors of transformation compounds were correlated with predicted ionization efficiencies of the same compounds. The predicted logarithmic response factors of analytes were obtained using Eq. 5:

$$
\log R F_{\text {pred }}=\text { Slope } \times \log I E_{\text {pred }}+\text { intercept }
$$

Eq. 5

where slope and intercept values are determined from the abovementioned correlation graph of logarithmic response factors of transformation compounds vs their predicted ionization efficiencies. As analysts are mainly interested in concentrations, the concentrations are calculated from predicted response factors of analytes according to Eq. 6 :

$$
c_{\text {pred }}=\frac{\mathrm{Signal} \cdot \mathrm{IC}}{10^{\log R F_{\text {pred }}}}
$$

Eq. 6

where Signal is MS1 peak area, and $I C$ is an isotopic correction, which is the sum of the abundances of all isotopomers for each compound. As a result, molar concentrations are obtained.

To estimate the accuracy of prediction the absolute times differences of spiked concentration and predicted concentration in both cereal matrices as well as in pure solvent were calculated as Eq 7.

$$
\text { Prediction error }=\max \left\{\begin{array}{l}
\frac{c_{\text {pred }}}{c_{\text {spiked }}} \\
\frac{c_{\text {spiked }}}{c_{\text {pred }}}
\end{array}\right.
$$

a

\section{RESULTS AND DISCUSSION}

\subsection{Predicting Ionization Efficiency Values}

The Quantem model (J. Liigand et al., 2019) developed previously, was used to predict the ionization efficiencies of the studied compounds. These values were transferred to instrument-specific response factors $(\log R F)$ with the aid of Eq 4 and good transformation accuracy was observed both on QQQ in $\mathrm{UT}\left(R^{2}=0.77\right)$ and QToF in DTU $\left(R^{2}=0.66\right)$. 
183

184

185

186

187

188

189

190

191

192

193

194

195

196

197

The transformation compounds are used for transferring the ionization efficiency values into instrument-specific values and should be measured under conditions as close as possible to the actual sample analysis. Therefore, it is of interest if the transformation compounds need to be spiked into the sample or can be analyzed as a separate mixture within the same analysis sequence. To test this transformation compounds were spiked into two matrices (barley and oat) and were run as a separate standard solution. The concentration prediction accuracy for 109 randomly selected pesticides were compared. It was seen that if the model transferring compounds were spiked into the matrices, the mean errors changed only slightly from 3.8 to 3.9 times, thus there is hardly any difference (Table S2). Therefore, it is feasible to transform the predicted ionization efficiency values by measuring the transformation compounds in the standard solution instead of spiking into specific matrices.

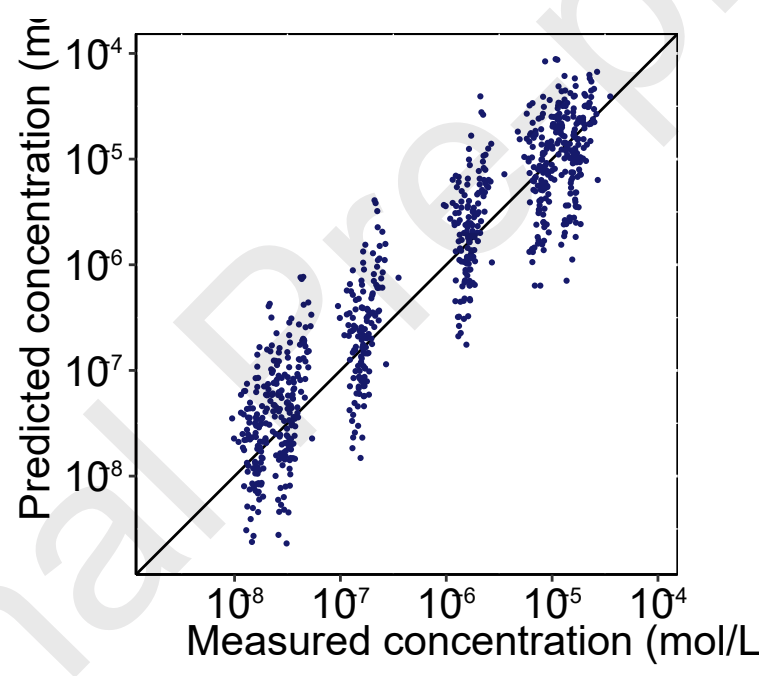

Figure 1 Correlation between the predicted and measured concentrations for the pesticides in solvent (QQQ in UT).

\subsection{Ionization Efficiency and concentration prediction in solvent and matrices.}

\subsubsection{Concentration prediction in a solvent on QQQ}


In order to determine the applicability of this Quantem model for a wider range of compounds, a set of pesticides and mycotoxins with varying chemical nature was chosen and measured. The studied set on QQQ includes 139 common pesticides from different compound classes, such as amide, anilide, ether, pyrimidine, ketone. Each pesticide was studied at 6 concentration levels covering more than 4 orders of magnitude in both matrices and solvent. The spiked concentrations ranged from $10 \mathrm{nM}$ to $35 \mu \mathrm{M}$. The MRL for pesticides in cereal samples is 0.01 to $0.02 \mathrm{ppm}$. This corresponds to a pesticide concentration of $\sim 100 \mathrm{nM}$, which is in the lower end of the studied concentration range.

The predicted $\log I E$ values (Table S1) for all the tested compounds varied from 1.3 to 4.5. From the prediction of concentration in pure solvents, we found that for $48 \%$ of compounds, the concentration prediction error is better than 2 times, for $87 \%$ of compounds better than 5 times, and for $98 \%$ of studied compounds better than 10 times. The highest prediction error of concentration was 16 times. The 3 bestperforming pesticides were imazamox, tetraconazole, and metrafenone (1.1 times for all). It is evident that the model is universal for both nitrogen as well as for oxygen bases, as among the top performers are both nitrogen and oxygen bases. Unsurprisingly, compounds (altogether 4 compounds, e.g., propiconazole) included previously in developing the $\log I E$ prediction model show high performance (J. Liigand et al., 2019). The correlation between measured and predicted concentration values in the solvent is presented in Figure 1; it can be seen that the agreement between measured and predicted concentration of test compounds by the model was good, the average accuracy was 3.0 times.

Comparing the different concentration levels, the accuracy was consistent except for the lowest concentration level, where it is only slightly lower (4.1 times).

\subsubsection{Concentration Prediction in Matrices on QQQ.}

The performance of Quantem model for prediction of $\log I E$ values for 6 cereal matrices was evaluated for the analyses of the same compounds at the same concentration levels as in solvent. Altogether, 6 concentration levels from $10 \mathrm{nM}$ to $35 \mu \mathrm{M}$ were used. The detail prediction error results in different matrices were shown in Table S3. It was found the mean errors of concentration mismatch between predicted and measured values in all cereal matrices was 3.8 times (Figure 2), the average concentration prediction error for each cereal matrix varied from 3.3 times to 4.6 times. In general, higher prediction accuracy was observed for wheat than for the other matrices (prediction error of 3.3 times), oat performed worst (4.6 times). Comparing all different concentration levels (Table S5), the prediction 
error of the lowest concentration level was slightly higher (4.4 times). For $46 \%$ of compounds, the concentration prediction error was less than 2 times, for $81 \%$ of compounds less than 5 times, and for $94 \%$ of compounds less than 10 times.

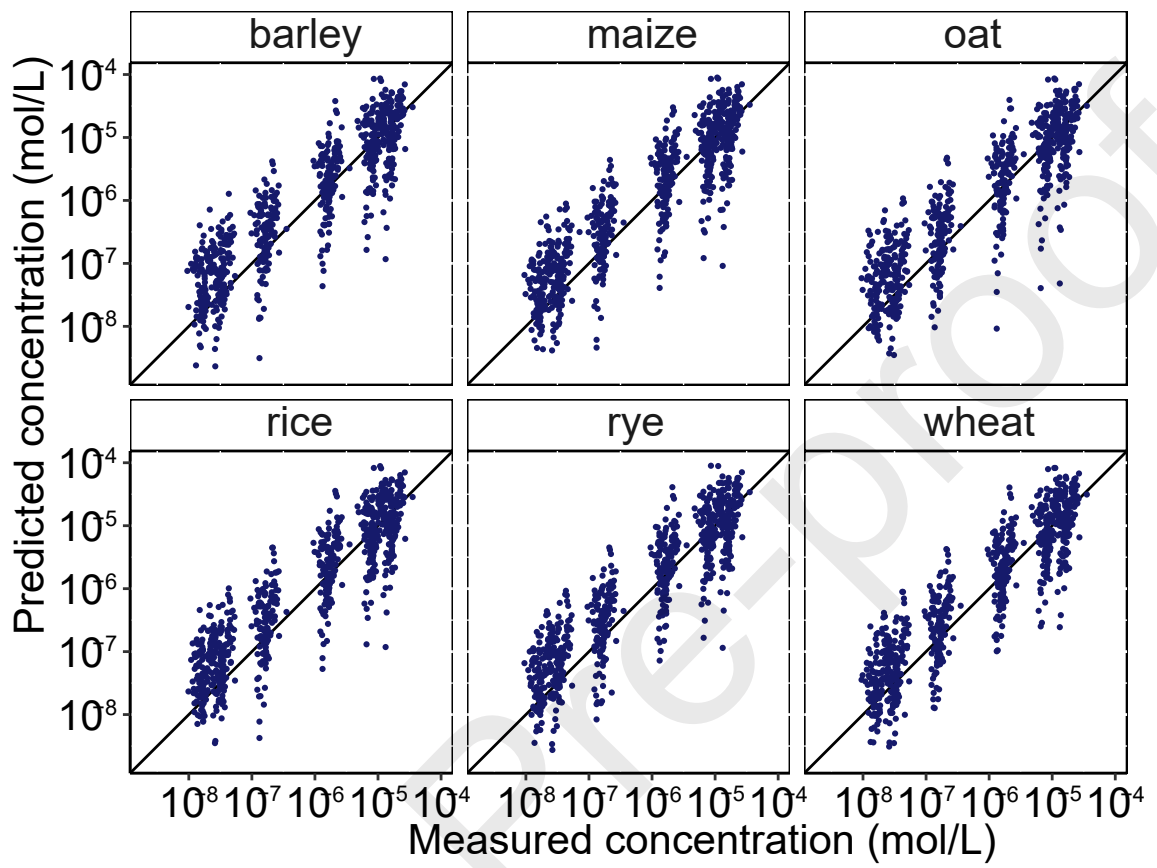

230

231

232

233

234

235

236

237

238

239

240

241

242

243

Figure 2 Correlation between the predicted and measured concentrations for pesticides in 6 cereal matric extracts. The measurements were performed on the QQQ instrument in UT.

The concentration prediction accuracy of carbosulfan was most severely influence by matrices (prediction error 64.6 times), which had the highest difference while compared to prediction accuracy in solvent (3.9 times). The reason of carbosulfan's poor performance could be that it contains the N-S-N moiety which was not present in the training set of the Quantem model. On the other hand, carbosulfan is unstable, it decomposes slowly at room temperature especially in acidic organic solvents (Barros \& Barros, 1995; Leppert, Markle, Helt, \& Fujie, 1983). The QuEChERS sample preparation used in this study uses $0.2 \%$ formic acid in the sample extraction procedure. We also observed that the degradation of carbosulfan results in poor linearity in matrices. It can be found that carbosulfan in oat matrice results in the largest concentration prediction error of 173.8 times. Carbosulfan was followed by fluometuron (15.9 times), pirimicarb (15.7 times), pyridaben (15.4 times), and pyridate (14.1 times) on average in all matrices. 


\subsubsection{Matrix effect}

The results are influenced by the matrix effect: the ionization suppression due to the matrix compounds co-eluting with the analyte. The most severe matrix effect was observed in oat matrix, the matrix effect was in the range of $2 \%$ (suppressed by $98 \%$ ) to $109 \%$ (enhanced by $9 \%$ ) for all compounds relative to the pure solvent (Table S7). In comparison to other cereals, oat is a rich source of polar lipids and free fatty acids. It has been discussed that phospholipids and free fatty acids will cause matrix effects in ESI (Krasilnikov, Batalova, Popov, \& Sergeyeva, 2018; Pucci, Di, Alfieri, Bonelli, \& Monteagudo, 2009), which can explain the severe matrix effect found in oat. The matrix effect may vary with analyte concentration (Furey, Moriarty, Bane, Kinsella, \& Lehane, 2013; Kruve, Auling, Herodes, \& Leito, 2011).

The concentration prediction errors were in general consistent for most compounds in both solvent and matrices, which means that the matrix effect does not influence the prediction accuracy significantly and the transformation step help to account for the matrix effects. For example, fluometuron, pyridaben, pirimicarb, pirimicarb-desmethyl, EPN, and thiamethoxam, had low prediction accuracy in both solvent and matrices. For some compounds, specific matrices caused a notable decrease in prediction accuracy. Carbosulfan and pyridate suffered the most severe matrix effects, carbosulfan was suppressed $98 \%$ and 95\% in oat and maize matrices (concentration prediction error of 173.8 times and 55.5 times), respectively. Signal of pyridate was suppressed $87 \%$ and $73 \%$ in oat and maize matrices (concentration prediction error of 20.3 times and 24.1 times), respectively. Signal of benfuracarb was suppressed 58\% in the oat matrix, which results in 5.9 times concentration prediction error. Spirodiclofen and pyridaben were also suppressed $53 \%$ and $47 \%$ in the matrices, resulted in concentration prediction error of 10.3 times and 15.4 times on average.

\subsubsection{Concentration Prediction in Matrices on QToF}

In order to test the robustness of the Quantem model and the transferability of the ionization efficiency prediction model between labs, the same matrices spiked at the 6 concentration levels from $4 \mathrm{nM}$ to $11 \mu \mathrm{M}$ with QToF in DTU. The ionization efficiency prediction and transformation approach were the same as in the case of QQQ instrument. There were 134 pesticides in common that could be 
detected and quantified both in DTU and UT. After model transformation, the predicted $\log I E$ values (Table S4) for all the tested compounds on QToF varied from 1.6 to 4.5.

As shown in Figure 3, the concentration prediction accuracy was even better on QToF in DTU. The average concentration prediction errors for all compounds was 2.4 times. For $61 \%$ of the compounds, the prediction error was better than 2 times and for $94 \%$ of compounds better than 5 times. These values are reasonably close to the ones obtained on the QQQ instrument in UT and described above. The average concentration prediction errors were 2.3 times in solvent and 2.6 times in all matrices. The prediction errors in different matrices were in the range from 2.5 to 3 times, which is quite closed. Rice and rye performed slightly better than other matrices. The concentration prediction errors were still in general consistent for most compounds in solvent and matrices. As the QToF instrument used for analysis in DTU is high-resolution mass spectrometry, the ability to distinguish the molecules with close MS weight is better compared to QQQ; also, the total running time for each sample in DTU is longer than the running time in UT which leads to a better separation on HPLC part. As a result, the integration of the compounds on QToF was influenced less by other compounds and the matrices background than QQQ. This might explain the slightly better performance of the concentration prediction for the data in DTU than UT.

On the whole, the quantitation of pesticides in cereal matrices with LC/ESI/MS without standard substances is feasible to achieve the prediction error of less than 4 times on average both on QToF in DTU and QQQ in UT. 


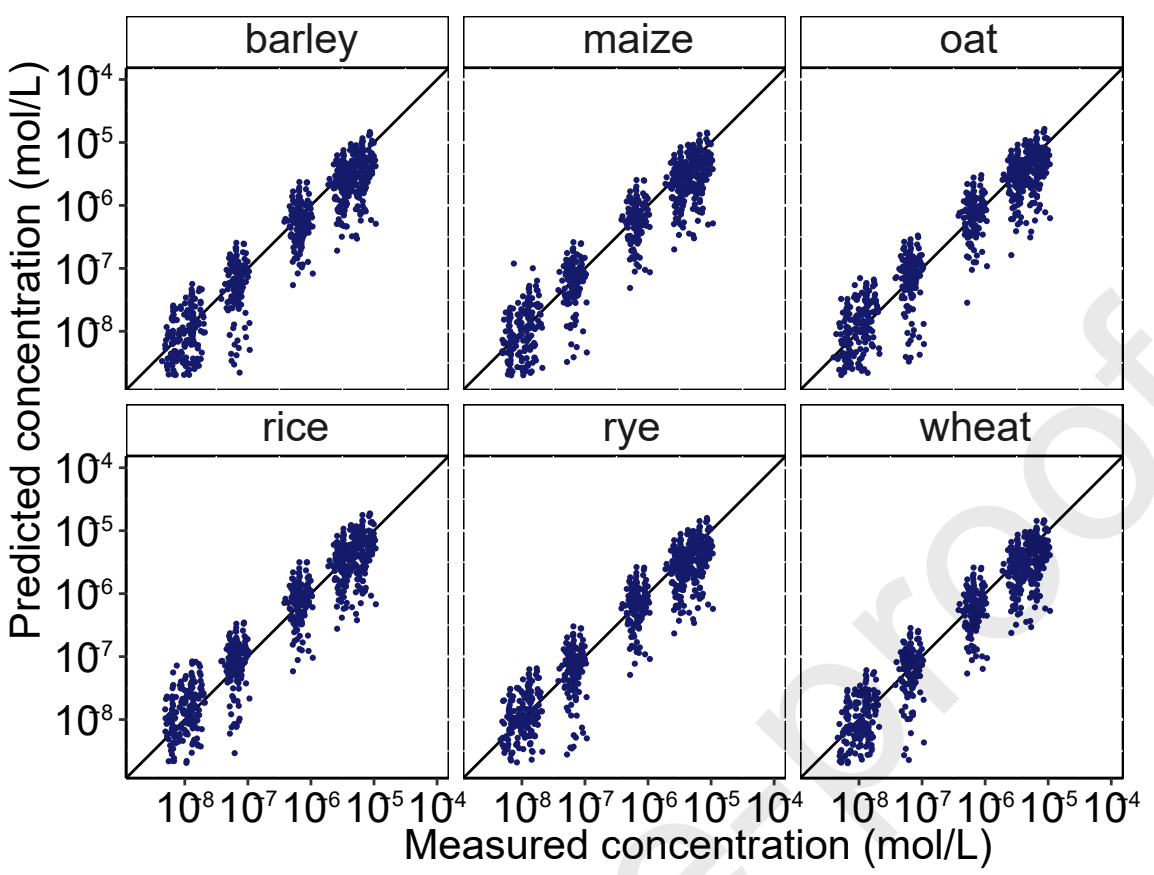

289

290

291

292

293

294

295

296

297

298

299

300

301

302

303

Figure 3 Correlation between the predicted and measured concentrations for pesticides in 6 cereal matric extracts. The measurements were performed on QToF instrument in DTU.

\subsubsection{Comparison of the predicted concentrations between labs.}

Moreover, after applying Quantem model we can also directly compare the concentrations obtained both in UT and DTU. The methods applied in UT and DTU are intrinsically different. These use slightly different chromatography, different mobile phase, different ESI source as well as different mass analyzers and vendors. As a result, the chromatograms obtained in both labs are not comparable. However, as the Quantem model translate these chromatograms into quantitative data for pesticides and the results can be numerically compared. Here we observed a very high correlation for the results from UT and DTU $\left(R^{2}=0.85\right)$. Also, the slope and intercept of the correlation line were very close to the ideal fit, the slope was 0.95 while the ideal would be 1. The results are shown in Figure 4. From this, it is obvious that for the first time the measurement results from two different laboratories can be made quantitatively comparable for suspect screening without the necessity of standard substances. This finding is extremely valuable for applications in both food monitoring and environmental areas. 


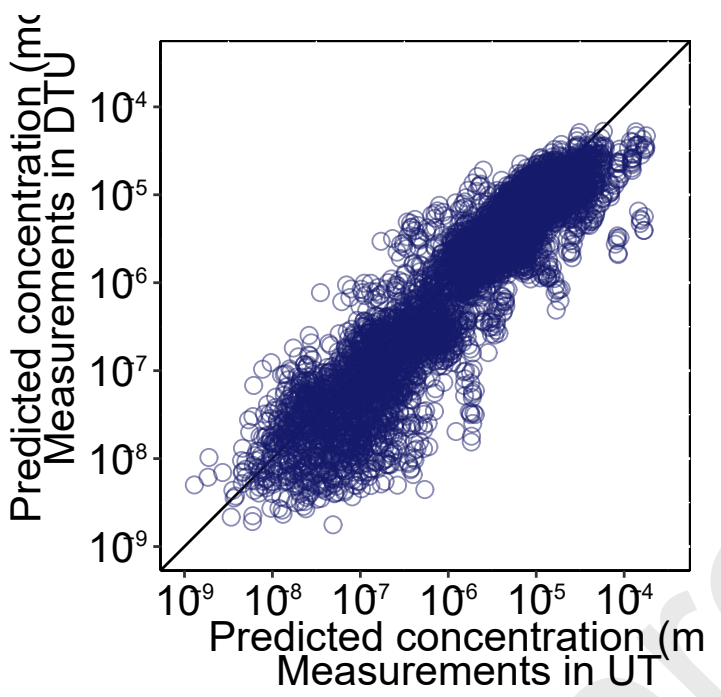

304

305 306

307

308

309

310

311

312

313

314

315

316

317

318

319

320

321

322

323

Figure 4 Correlation between the predicted concentrations for pesticides in 6 cereal matrices measured on QQQ at UT and on QToF at DTU.

\subsubsection{LoD and LoQ}

In spite of the generally good correlation between the predicted concentrations for the analysis of two different labs (UT and DTU), some of the compounds were detected only by one of the labs. In the case of interlaboratory comparisons, such differences often occur and commonly indicate different LoD and LoQ values. Therefore, we compared the estimated concentrations of the compounds detected by both labs with the concentrations of the compounds detected only in DTU with QToF instrument. The comparison revealed that most of the compounds not detected by UT were having among some of the lowest concentrations (Figure 5). Therefore, the comparison for the concentration determined from different labs has the potential to bring clarity to the discrepancies often observed in collaborative trials of non-targeted screening.

To further validate the conclusion, we determine the LoD values of all of the studied compounds. This was possible as the concentrations of the pesticides were known from the spiking experiments. A conservative LoD determination strategy was used (Evard et al., 2016). It was observed that the LoD values ranged between $0.1 \mathrm{nM}$ and $1 \mu \mathrm{M}$, while most values were between $1 \mathrm{nM}$ and $0.1 \mu \mathrm{M}$. The LoD and LoQ values are brought in Table S9. The comparison of the determined LoD values for UT and DTU, in Figure S1, revealed that different compounds had varying LoD values between different labs. Sometimes the LoD was lower in UT, sometimes in DTU. 


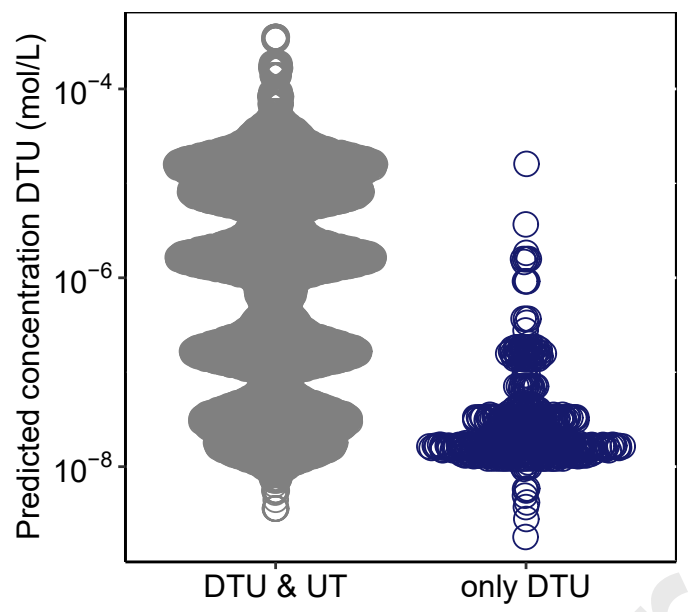

324

325 Figure 5 The predicted concentrations of pesticides for compounds detected in both laboratories vs 326 detected only in DTU. The results are summarized over all studied matrices.

327 


\section{Conclusion}

Here we have shown, for the first time that results obtained from suspect screening can be made quantitatively comparable between different laboratories using different instruments as well as different analysis methods. To achieve this, we use the ionization efficiency prediction with Quantem model to transfer the obtained signals into concentrations. In contrast to signals, the concentrations are comparable between different instruments and labs. The consistency between the results obtained independently in two labs was very high $\left(R^{2}=0.85\right)$ in spite of the differences in the instrumentation and methods. Also, the independent results show high accuracy. The quantitation of pesticides in different cereals matrices without standard substances is feasible with the accuracy of less than 4 times on average for chromatographic analyses. It can serve as a useful tool for preliminary quantitation in various fields such as food control, metabolomics, and environmental research, and there is a significant improvement compared to the conventional approach. Also, the obtained accuracy is sufficient for using the standard substance-free quantitation as the first step in food monitoring.

\section{SUPPORTING INFORMATION DESCRIPTION}

342 Supplementary data related to this article can be found in the Supporting Information file.

\section{ACKNOWLEDGEMENTS}

344 This work was financially supported by Smart specialization doctoral stipend, PRG300 from Estonian 345 Research Council, Otto Mønsted Foundation, and Fødevareforlig 3. To all financing sources the authors 346 are greatly indebted. 


\section{REFERENCE}

349 Alymatiri, C. M., Kouskoura, M. G., \& Markopoulou, C. K. (2015). Decoding the signal response of steroids in electrospray ionization mode (ESI-MS). Analytical Methods, 7, 10433-10444. https://doi.org/10.1039/C5AY02839F

352

353

354

355

356

357

358

359

360

361

362

Barros, M. W., \& Barros, A. (1995). Determination of Carbosulfan in Oranges by High-performance Liquid Chromatography With Post-col umn Fluorescence, 120, 2479-2481.

Cramer, C. J., Johnson, J. L., \& Kamel, A. M. (2017). Prediction of Mass Spectral Response Factors from Predicted Chemometric Data for Druglike Molecules. Journal of the American Society for Mass Spectrometry, 28, 278-285. https://doi.org/10.1007/s13361-016-1536-4

Dong, J., Cao, D. S., Miao, H. Y., Liu, S., Deng, B. C., Yun, Y. H., ... Chen, A. F. (2015). ChemDes : an integrated web - based platform for molecular descriptor and fingerprint computation. Journal of Cheminformatics, 7(60), 1-10. https://doi.org/10.1186/s13321-015-0109-z

Dzuman, Zbynek, Zachariasova, Milena, Veprikova, Z., Godula, M., \& Hajslova, J. (2015). Multianalyte high performance liquid chromatography coupled to high resolution tandem mass spectrometry method for control of pesticide residues, mycotoxins, and pyrrolizidine alkaloids. Analytica Chimica Acta, 863(1), 29-40. https://doi.org/10.1016/j.aca.2015.01.021

EU - Pesticides database. (n.d.). Retrieved from http://ec.europa.eu/food/plant/pesticides/eu-pesti Evard, H., Kruve, A., \& Leito, I. (2016). Tutorial on estimating the limit of detection using LC-MS analysis, part I: Theoretical review. Analytica Chimica Acta, 942, 23-39. https://doi.org/10.1016/j.aca.2016.08.043

Fraser, K., Lane, G. A., Otter, D. E., Harrison, S. J., Quek, S. Y., Hemar, Y., \& Rasmussen, S. (2014). Non-targeted analysis by LC-MS of major metabolite changes during the oolong tea manufacturing in New Zealand. Food Chemistry, 151, 394-403. https://doi.org/10.1016/j.foodchem.2013.11.054

Furey, A., Moriarty, M., Bane, V., Kinsella, B., \& Lehane, M. (2013). Ion suppression ; A critical review on causes, evaluation, prevention and applications. Talanta, 115, 104-122. https://doi.org/10.1016/j.talanta.2013.03.048

Fusaro, V. A., Mani, D. R., Mesirov, J. P., \& Carr, S. A. (2009). Prediction of high-responding peptides 
for targeted protein assays by mass spectrometry. Nature Biotechnology, 27(2), 190-198. https://doi.org/10.1038/nbt.1524

Golubović, J., Birkemeyer, C., Protić, A., Otašević, B., \& M., Z. (2016). Structure - response relationship in electrospray ionization-mass spectrometry of sartans by artificial neural networks. Journal of Chromatography A, 1438, 123-132. https://doi.org/10.1016/j.chroma.2016.02.021

González-Curbelo, M. Á., Herrera-Herrera, A. V, Ravelo-Pérez, L. M., \& Hernández-Borges, J. (2012). Sample-preparation methods for pesticide-residue analysis in cereals and derivatives. Trends in Analytical Chemistry, 38, 32-51. https://doi.org/10.1016/j.trac.2012.04.010

Hermans, J., Ongay, S., Markov, V., \& Bischoff, R. (2017). Physicochemical Parameters Affecting the Electrospray Ionization Efficiency of Amino Acids after Acylation. Analytical Chemistry, 89, 9159-9166. https://doi.org/10.1021/acs.analchem.7b01899

Hird, S. J., Lau, B. P.-Y., Schuhmacher, R., \& Krska, R. (2014). Liquid chromatography-mass spectrometry for the determination of chemical contaminants in food. TrAC Trends in Analytical Chemistry, 59, 59-72. https://doi.org/10.1016/j.trac.2014.04.005

Huffma, B. A., Poltash, M. L., \& Hughey, C. A. (2012). Effect of Polar Protic and Polar Aprotic Solvents on Negative-Ion Electrospray Ionization and Chromatographic Separation of Small Acidic Molecules. Analytical Chemistry, 84, 9942-9950. https://doi.org/10.1021/ac302397b

Jamin, E. L., Bonvallot, N., \& Tremblay-franco, M. (2014). Untargeted profiling of pesticide metabolites by LC - HRMS : an exposomics tool for human exposure evaluation. Anal Bioanal Chem, 406, 1149-1161. https://doi.org/10.1007/s00216-013-7136-2

Kalogiouri, N. P., Aalizadeh, R., \& Thomaidis, N. S. (2017). Investigating the organic and conventional production type of olive oil with target and suspect screening by LC-QTOF-MS, a novel semi-quantification method using chemical similarity and advanced chemometrics. Analytical and Bioanalytical Chemistry, 49(3), 5413-5426. https://doi.org/10.1007/s00216-0170395-6

Krasilnikov, V. N., Batalova, G. A., Popov, V. S., \& Sergeyeva, S. S. (2018). Fatty Acid Composition of Lipids in Naked Oat Grain of Domestic Varieties. Russian Agricultural Sciences, 44(5), 406408. https://doi.org/10.3103/S1068367418050117

Kruve, A., Auling, R., Herodes, K., \& Leito, I. (2011). Study of liquid chromatography / electrospray 
ionization mass spectrometry matrix effect on the example of glyphosate analysis from cereals. Rapid Commun. Mass Spectrom, 25, 3252-3258. https://doi.org/10.1002/rcm.5222

Kruve, A., \& Kaupmees, K. (2017). Predicting ESI/MS signal change for anions in different solvents. Analytical Chemistry, 89, 5079-5086. https://doi.org/10.1021/acs.analchem.7b00595

Kruve, A., Kaupmees, K., Liigand, J., \& Leito, I. (2014). Negative electrospray ionization via deprotonation: Predicting the ionization efficiency. Analytical Chemistry, 86(10), 4822-4830. https://doi.org/10.1021/ac404066v

Leppert, B. C., Markle, J. C., Helt, R. C., \& Fujie, G. H. (1983). Determination of Carbosulfan and Carbofuran Residues in Plants, Soil , and Water by Gas Chromatography. J. Agrie. Food Chem, 31, 220-223. https://doi.org/10.1021/jf00116a009

Li, Z., \& Jennings, A. (2017). Worldwide Regulations of Standard Values of Pesticides for Human Health Risk Control : A Review. International Journal of Environmental Research and Public Health, 14, 826. https://doi.org/10.3390/ijerph14070826

Liigand, J., Wang, T., Kellogg, J., Smedsgaard, J., Cech, N., \& Kruve, A. (2019). Quantifying the unquantifiable: Quantification for non-targeted LC/MS screening without standards, submitted.

Liigand, P., Liigand, J., Cuyckens, F., Vreeken, R. J., \& Kruve, A. (2018). Ionisation efficiencies can be predicted in complicated biological matrices: A proof of concept. Analytica Chimica Acta, 1032, 68-74. https://doi.org/10.1016/j.aca.2018.05.072

Mol, H. G. J., Plaza-Bolaños, P., Zomer, P., De Rijk, T. C., Stolker, A. A. M., \& Mulder, P. P. J. (2008). Toward a generic extraction method for simultaneous determination of pesticides, mycotoxins, plant toxins, and veterinary drugs in feed and food matrixes. Analytical Chemistry, 80(24), 9450-9459. https://doi.org/10.1021/ac801557f

Oss, M., Kruve, A., Herodes, K., \& Leito, I. (2010). Electrospray ionization efficiency scale of organic compound. Analytical Chemistry, 82, 2865-2872. https://doi.org/10.1021/ac902856t

Pieke, E. N., Granby, K., Trier, X., \& Smedsgaard, J. (2017). A framework to estimate concentrations of potentially unknown substances by semi-quantification in liquid chromatography electrospray ionization mass spectrometry. Analytica Chimica Acta, 975, 30-41. https://doi.org/10.1016/j.aca.2017.03.054

Pucci, V., Di, S., Alfieri, A., Bonelli, F., \& Monteagudo, E. (2009). A novel strategy for reducing 
457 458 459

460 461

phospholipids-based matrix effect in LC-ESI-MS bioanalysis by means of HybridSPE. Journal of Pharmaceutical and Biomedical Analysis, 50, 867-871. https://doi.org/10.1016/j.jpba.2009.05.037

Raji, M. A., Fryčák, P., Temiyasathit, C., Kim, S. B., Mavromaras, G., Ahn, J. -., \& Schug, K. A. (2009). Using multivariate statistical methods to model the electrospray ionization response of GXG tripeptides based on multiple physicochemical parameters. Rapid Communications in Mass Spectrometry, 23, 2221-2232. https://doi.org/10.1002/rcm

Wang, T., Frandsen, H. L., Christiansson, N. R., Rosendal, S. E., Pedersen, M., \& Smedsgaard, J. (2019). Pyrrolizidine alkaloids in honey: Quantification with and without standards. Food Control, 98, 227-237. https://doi.org/10.1016/j.foodcont.2018.11.033

Wu, L., Wu, Y., Shen, H., Gong, P., Cao, L., Wang, G., \& Hao, H. (2013). Quantitative structure-ion intensity relationship strategy to the prediction of absolute levels without authentic standards. Analytica Chimica Acta, 794, 67-75. https://doi.org/10.1016/j.aca.2013.07.034

Yap, C. W. (2011). Software News and Update PaDEL-Descriptor : An Open Source Software to Calculate Molecular Descriptors and Fingerprints. Journal of Computational Chemistry, 32(7), 1466-1474. https://doi.org/10.1002/jcc

T.W. carried out all the measurements, planned the experiments and wrote a big part of the manuscript. J.L. carried out all the ionization efficiency modelling and concentration prediction and helped writing the manuscript. J.S. and H.L.F. helped to plan and analyze data related to pesticide analysis. A.K. designed the project and helped to plan the experiments and modelling and helped writing the manuscript.

\section{Declaration of interests}

$\bigotimes$ The authors declare that they have no known competing financial interests or personal relationships that could have appeared to influence the work reported in this paper.

$\square$ The authors declare the following financial interests/personal relationships which may be considered as potential competing interests: 
462

463

464

465

466

467

468 Highlights

469 Six cereal matrices containing 134 pesticides (10 $\mathrm{nM}$ to $35 \mu \mathrm{M})$ were analysed

470 Measurements were done on $Q Q Q$ and ToF instruments in two independent laboratories

471 Pesticide are quantified with predicted electrospray ionization efficiencies

472 Quantitative results obtained in two labs were consistent with the $R^{2}$ of 0.85

473 